\title{
New Observations from the MiniBooNE Experiment
}

\author{
Geoffrey B. Mills ${ }^{1}$ \\ Los Alamos National Laboratory \\ Los Alamos, NM, USA \\ E-mail: millselanl.gov \\ For the MiniBooNE Collaboration
}

The MiniBooNE experiment has operated at Fermilab since 2002. It has collected $6.46 \times 10^{20}$ pot in neutrino mode and $5.66 \times 10^{20}$ pot in antineutrino mode. This paper discusses the most recent results of analyzing this data for oscillation like phenomena. An unexplained excess of $20.9 \pm 14.0$ events is observed in the latest electron-like antineutrino data. When a likelihood fit to a naïve 2-neutrino-oscillation appearance hypothesis is performed, it prefers an oscillation hypothesis, over the background-only hypothesis, with a significance probability of $99.5 \%(2.7$ $\sigma)$. The analysis of the data is still limited by low statistical precision, and the MiniBooNE experiment is foreseen to continue operating in antineutrino mode. The oscillation data is compared directly to the old result from LSND as a function of L/E, of relevance to neutrino oscillations.

35th International Conference of High Energy Physics (ICHEP2010)

Paris,France

July 22-28, 2010

1 Speaker 


\section{Introduction}

The MiniBooNE experiment has collected $6.46 \times 10^{20}$ protons-on-target (pot) in neutrino mode, and $5.66 \times 10^{20}$ pot in antineutrino mode through April 2010. The MiniBooNE detector is described elsewhere[2] and the prediction of the neutrino fluxes at the MiniBooNE detector is discussed in [3]. MiniBooNE has previously reported results on oscillation searches [4] and this paper reports on the analysis described in [5]. The first section briefly reminds the reader of the Fermilab booster neutrino beam (BNB), the MiniBooNE apparatus, the reconstruction and characteristics of the MiniBooNE data, and results reported previously. The next section describes the characteristics, the analysis, and analysis results of the MiniBooNE antineutrino data.

\section{Neutrino Beam, Detector, Event Reconstruction, and Event Selection}

The Fermilab $8 \mathrm{GeV}$ booster accelerator provides $1.6 \mu$ s long pulses of protons at an average frequency of $1-5 \mathrm{~Hz}$ to the BNB. Those proton pulses are guided to a beryllium target of 1.75 interaction lengths, and then interact to produce pions and kaons, which subsequently decay to neutrinos. Focusing the charged pions and kaons forward with a pulsed, toroidal field, magnetic horn, which operates at 174 kilo-amperes, enhances the forward flux of neutrinos by roughly a factor of five. The p-Be pion production cross sections at $8 \mathrm{GeV}$ proton energies were measured [6] and used to predict the neutrino flux. The muons, pions, and kaons are allowed to decay into neutrinos in a 50-meter long by 2-meter diameter decay tunnel, and the neutrinos then travel the 500 meter distance to the detector.

The spherical MiniBooNE detector tank is filled with 850 tons of mineral oil, which is viewed by 12508 -in photomultiplier tubes. Cerenkov light, from the relativistic byproducts of neutrino-interactions in the tank, creates specific patterns of light on the PMTs that allow for the discrimination of underlying event types by special software algorithms. With a mean neutrino energy of around $750 \mathrm{MeV}$, the MiniBooNE data is dominated by charged-current quasi elastic scattering (CCQE), $\Delta$ resonance production (CCPi), neutral-current, quasi elastic scattering (NCQE), and $\mathrm{NC} \pi^{0}$ production (NCPi0).

In order to select electron-like (e-like) events from $v_{\mathrm{e}}$ and $\bar{v}_{\mathrm{e}}$ interactions the following criteria were required: beam triggers with just one sub-event inside the $1.6 \mu$ s beam window; a reconstructed vertex radius $<500 \mathrm{~cm}$; the number of hit tank PMTs $>200$ and veto PMT hits $<$ 6. The resulting events are reconstructed according to four separate hypotheses: a single electron shower fit (e-fit), a single muon track fit ( $\mu$-fit), a two ring event fit (2ring-fit), and a $\pi^{0}$-fit (2-rings constrained to the $\pi^{0}$ mass). The e-like events are then selected by requiring the ratios of the fit likelihoods, e-fit $/ \mu$-fit and e-fit $/ \pi^{0}$-fit, to be large by the use of an energy dependent cut, and to require that the 2ring-fit reconstructed invariant mass be less than $50 \mathrm{MeV}$ (recall the $\pi^{0}$ mass is $135 \mathrm{MeV}$ ). Finally the visible energy is required to be greater than 140 $\mathrm{MeV}$, and an energy-dependent fiducial volume cut on inward-pointing events is made to remove the byproducts of neutrino interactions occurring outside the fiducial volume. 
A number of constraints on the $v_{\mathrm{e}}$ and $\bar{v}_{\mathrm{e}}$ backgrounds are derived directly from MiniBooNE data: the observed $\mathrm{NC} \pi^{0}$ rate is used to constrain background processes related to $\Delta$ production, for example radiative $\Delta \rightarrow \mathrm{N} \gamma$ decay; a sample of $\mu$-like CCQE events is used as a constraint on the intrinsic $v_{\mathrm{e}}$ and $\bar{v}_{\mathrm{e}}$ charged current processes; and events near the edge of the tank serve to normalize the number of events resulting from neutrino interactions occurring outside the tank. The resulting error on the expected e-like event rate after internal constraints was roughly $10 \%$, which included flux, cross section, and detector response systematic errors.

\section{Interpretation of the data}

The above event selection results in a sample of 277 events in the energy range between $200 \mathrm{MeV}$ and $3000 \mathrm{MeV}$, with an expected background of $233.8 \pm 15.3 \pm 16.5$ resulting in a modest excess of $43.2 \pm 22.5$ events. Since this data is also anti-neutrino appearance data in the $\mathrm{L} / \mathrm{E}$ range of the LSND result, it interesting to compare their respective measured oscillation probabilities as a function of $\mathrm{L} / \mathrm{E}$, as shown in Figure 1.

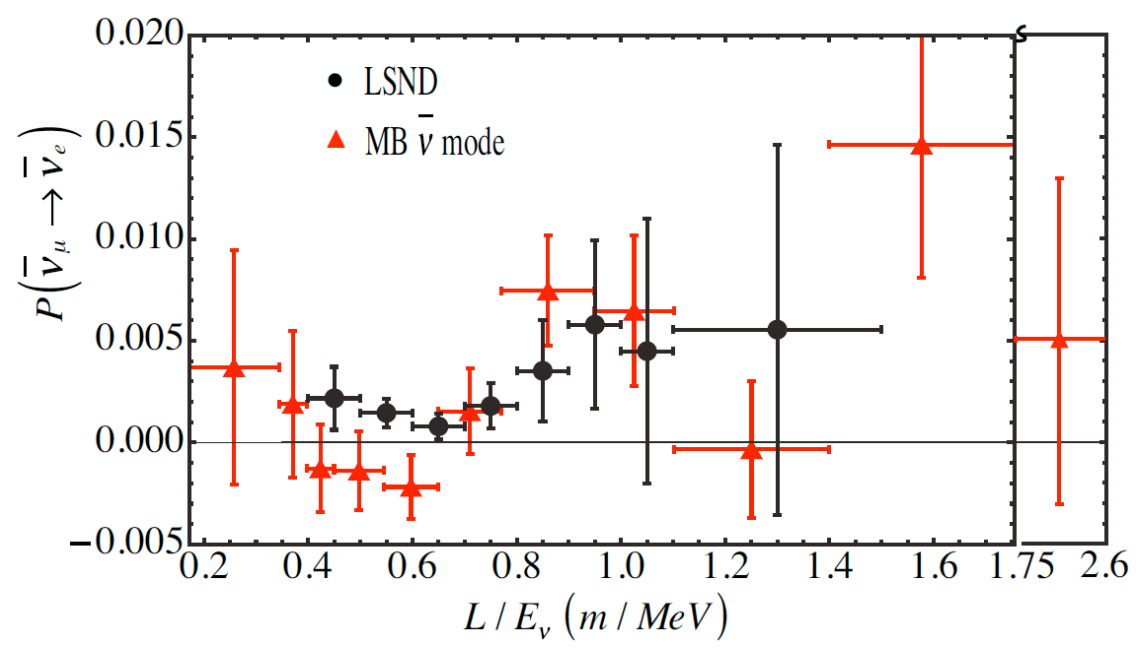

Figure 1 A comparison of the latest MiniBooNE and LSND $\bar{v}_{e}$ data as a function of L/E.

While the MiniBooNE data's precision is limited by its low statistics, it does exhibit variations in $\mathrm{L} / \mathrm{E}$ that are consistent with the hypothesis that the LSND excess is due to oscillations. To test the strength of the oscillation hypothesis, a likelihood fit to the e-like and $\mu$ like CCQE samples was used. Figure 2 shows the result of the fit was that the MiniBooNE data, when the background-only (i.e. null) hypothesis assumed, was disfavored at the 99.5\% CL (2.75 $\sigma)$. This was somewhat surprising, since the best-fit oscillation probability was substantially higher that the LSND best-fit value, however the MiniBooNE best-fit value is consistent with an upward statistical fluctuation from the LSND-best fit value.

The data excess shows some promise of being interpretable as an oscillation effect, but real proof can only come with MiniBooNE data at a different location in the beam line, for example a near detector run at 200 meters from the target. A study is currently underway to ascertain the best approach to take in order to accomplish that run. 

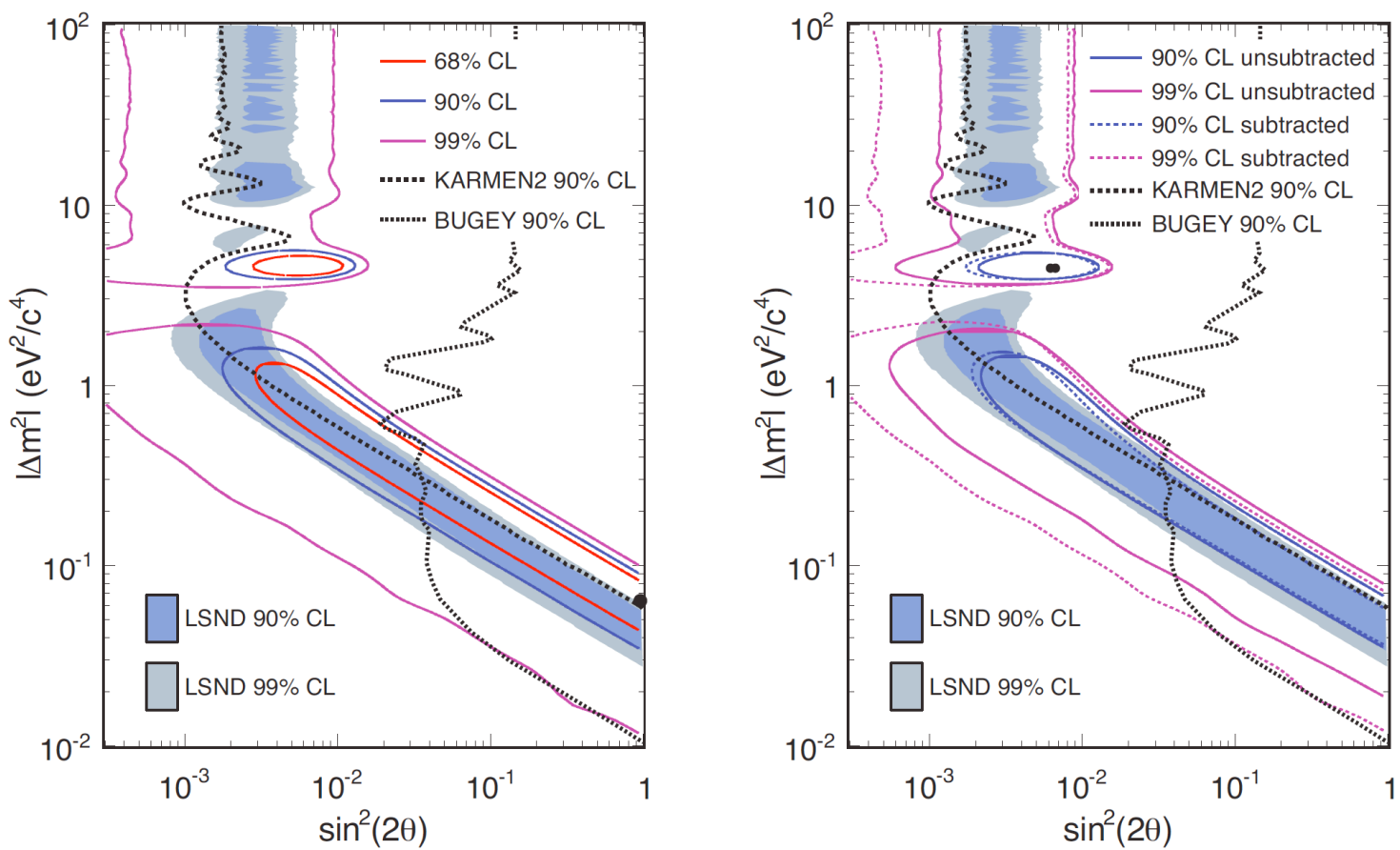

Figure 2 The result of performing a likelihood fit to the MiniBooNE data in the range from $475 \mathrm{MeV}$ (left) or $200 \mathrm{MeV}$ (right) to $3000 \mathrm{MeV}$. Also shown is the KARMEN[7] exclusion region, the Bugey[8] exclusion region, and the LSND allowed region. There are substantial regions of the LSND allowed regions that overlap with the new MiniBooNE antineutrino data, and which are not excluded by other experiments.

\section{References}

[1] A. A. Aguilar-Arevalo et al. [The LSND Collaboration], Phys. Rev. D64 , 112007 (2001)

[2] A. A. Aguilar-Arevalo et al. [The MiniBooNE Collaboration], NIM A599, 28-46 (2009)

[3] A. A. Aguilar-Arevalo et al. [The MiniBooNE Collaboration], Phys. Rev. D79, 072002 (2009)

[4] A. A. Aguilar-Arevalo et al. [The MiniBooNE Collaboration], Phys. Rev. Lett. 98, 231801 (2007);

A. A. Aguilar-Arevalo et al. [The MiniBooNE Collaboration], Phys. Rev. D78, 012007 (2008);A.

A. Aguilar-Arevalo et al. [The MiniBooNE Collaboration], Phys. Rev. Lett. 102, 101802 (2009);

A. A. Aguilar-Arevalo et al. [The MiniBooNE Collaboration], Phys. Rev. Lett. 103, 061802 (2009);

A. A. Aguilar-Arevalo et al. [The MiniBooNE Collaboration], Phys. Rev. Lett. 103, 111801 (2009)

[5] A. A. Aguilar-Arevalo et al. [The MiniBooNE Collaboration], Phys. Rev. Lett. 105, 181801 (2010)

[6] M. G. Catanesi et al. [The HARP Collaboration], Eur. Phys. J. C52, 29 (2007)

[7] B. Armbruster et al., Phys. Rev. D65, 112001 (2002)

[8] B. Achkar et al., Nucl. Phys. B434, 503 